\title{
Aerosol box, An Operating Room Security Measure in COVID-19 Pandemic
}

\author{
Francisco Alberto Leyva Moraga ${ }^{1}$ Eduardo Leyva Moraga ${ }^{1} \cdot$ Fernando Leyva Moraga $^{1}$. \\ Abelardo Juanz González ${ }^{2} \cdot$ Jesús Martín Ibarra Celaya $^{3} \cdot$ Jesús Antonio Ocejo Gallegos $^{1}$. \\ Jorge Arturo Barreras Espinoza ${ }^{2}$
}

Published online: 26 April 2020

(c) Société Internationale de Chirurgie 2020

Intubating and extubating patients in the operating room represents a high risk of exposure to aerosol droplets. Personal protective equipment must be utilized to protect surgical staff during the current COVID-19 outbreak. As the availability of protective gear diminishes in many healthcare settings, new reusable equipment is urgently needed. This situation prompted us to implement a new improvised barrier, denominated "Aerosol Box" (AB).

Originally based on Dr. Lai's design [1], we replicate a $50 \mathrm{~cm} \times 50 \mathrm{~cm} \times 40 \mathrm{~cm}$ polycarbonate sheet AB (Fig. 1), a suitable material to provide a transparent structure. This device is reusable, easy to produce, and resistant to high temperatures [2], with an approximate cost of \$40 USD per box. The $\mathrm{AB}$ allows the physician to cover the patient's head and manually undergo endotracheal intubation (Video1) and extubation (Video2) through two circular box openings, hence reducing the area of contact to expelled aerosol particles during the procedure [3]
Upon our experience with five patients, we encountered minor difficulties limiting the physician's range of motion during the procedure, although not cumbersome enough to halt laryngoscopy maneuvers or impede endotracheal tube placement. We recommend performing video laryngoscopy-assisted endotracheal intubation by an experienced anesthesiologist, previously utilizing training manikins; however, standard laryngoscopy can be performed if the video laryngoscope is not available. It is imperative to remove the $\mathrm{AB}$ if it represents an impediment to the healthcare provider during the procedure sequence.

Maneuvers to support the physician during the procedure can be done by introducing the assistant's hand through the open side of the AB. These maneuvers include applying cricoid pressure, securing the endotracheal tube and removing the guide wire, while simultaneously attempting to intubate the patient under the covered area by
Electronic supplementary material The online version of this article (https://doi.org/10.1007/s00268-020-05542-x) contains supplementary material, which is available to authorized users.

Eduardo Leyva Moraga

leyvamoragaeduardo@gmail.com

1 Department of Medicine and Health Science, Universidad de Sonora, Av Luis Donaldo Colosio, 83000 Hermosillo, México

2 Surgery Department, Hospital General del Estado de Sonora, Hermosillo, México

3 Thoracic Surgery Deparment, Hospital +Querétaro, Santiago de Querétaro, Querétaro, México 


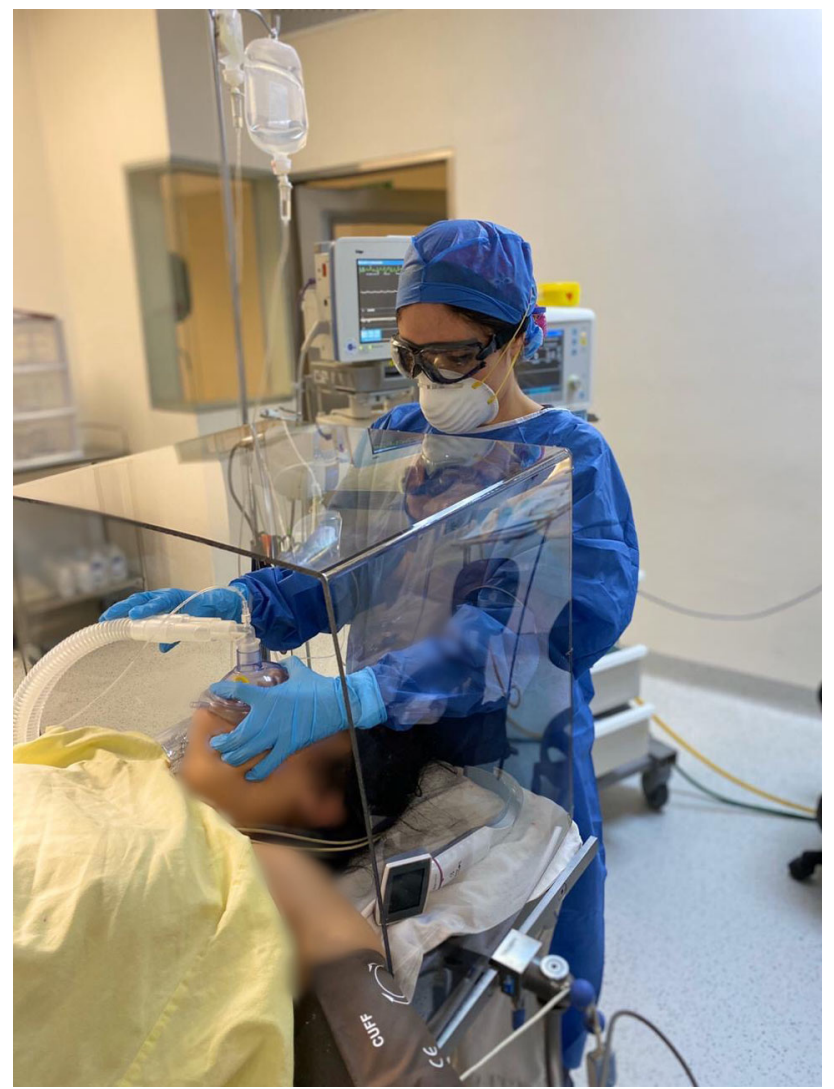

Fig. 1 Colocation of Aerosol Box

the $\mathrm{AB}$. To reuse the $\mathrm{AB}$, we cleaned the surface areas with $5000 \mathrm{mg} / \mathrm{L}$ bleach.

The world is facing an outstanding sanitary crisis and we, as the healthcare system's frontline force, are fighting to provide the highest quality of care to our patients while protecting our safety. This challenge represents an enormous responsibility, held by a commitment to our community and ourselves.

Our AB model has proven to be a valuable resource in our center, functioning as an adaptive tool to aid in our resource-limiting setting. The $\mathrm{AB}$ did not represent an obstacle to our established protocol, and we believe it can be one of the eagerly sought solutions in low- and middleincome healthcare settings facing this pandemic.

Acknowledgments We thank Dr. Luigi Pellegrini and Dr. Fernanda Covarrubias for providing the videos and image illustrated in this paper.

\section{Compliance with ethical standards}

Conflict of interest The authors certify that they have no involvement in any organization with any financial or non-financial interest, in the subject matter discussed in the manuscript.

\section{References}

1. Lai HY, Design A (2020) Aerosol box-design. [online] Sites.google.com. Available at: <https://sites.google.com/view/aerosol box/design $>$ (Accessed 29 March 2020)

2. Maddah HA (2016) Polypropylene as a promising plastic: a review, American. J Polymer Science 6(1):1-11. https://doi.org/10. 5923/j.ajps.20160601.01

3. Canelli R, Connor C, Gonzalez M, Nozari A, Ortega R (2020) Barrier enclosure during endotracheal intubation. N Engl J Med. https://doi.org/10.1056/NEJMc2007589

Publisher's Note Springer Nature remains neutral with regard to jurisdictional claims in published maps and institutional affiliations. 